\title{
ARM Value-Added Product (VAP) Monthly Status Report
}

\author{
ARM Translator Team \\ J. Comstock \\ C. Flynn \\ M. Jensen \\ C. Long \\ D. Turner \\ S. Xie
}

March 13, 2007

Work supported by the U.S. Department of Energy, Office of Science, Office of Biological and Environmental Research 


\section{DISCLAIMER}

This report was prepared as an account of work sponsored by the U.S. Government. Neither the United States nor any agency thereof, nor any of their employees, makes any warranty, express or implied, or assumes any legal liability or responsibility for the accuracy, completeness, or usefulness of any information, apparatus, product, or process disclosed, or represents that its use would not infringe privately owned rights. Reference herein to any specific commercial product, process, or service by trade name, trademark, manufacturer, or otherwise, does not necessarily constitute or imply its endorsement, recommendation, or favoring by the U.S. Government or any agency thereof. The views and opinions of authors expressed herein do not necessarily state or reflect those of the U.S. Government or any agency thereof. 


\section{Contents}

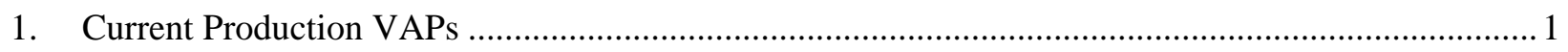

1.1 Atmospherically Emitted Radiance Interferometer (AERI) Noise Filter.................................. 1

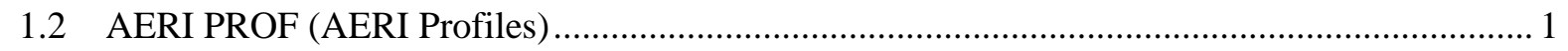

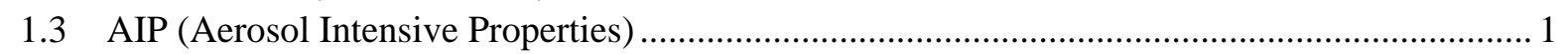

1.4 AOS Fit RH (Aerosol Observing System Fit RH) ............................................................ 2

1.5 ARSCL (Active Remote Sensing of Cloud Layers) …......................................................... 2

1.6 BAEBBR (Bulk Aerodynamics Energy Balance Bowen Ratio)............................................. 2

1.7 BEFLUX (Best-estimate Surface Radiative Flux) ................................................................... 3

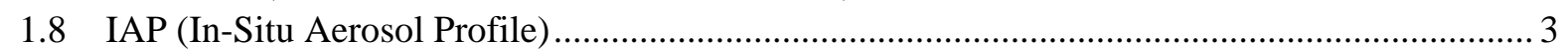

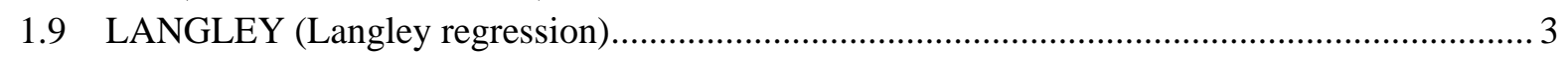

1.10 LBL CLOUD E (Line-by-Line Cloud Emissivity) …............................................................. 3

1.11 LBL RTM AERI (Line-by-Line Radiative Transfer Model to match AERI) ............................. 3

1.12 LBL MWR (Line-by-line calculations compared to the MWR) ............................................... 4

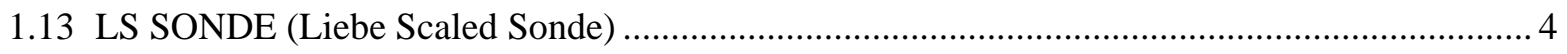

1.14 MFRSR CLDOD (Cloud Optical Properties) ..................................................................... 4

1.15 MFRSR OD (MFRSR Optical Depths) ….......................................................................... 4

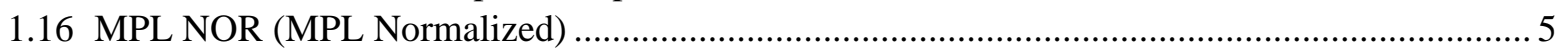

1.17 MWR AVG (Microwave Radiometer Averaging)................................................................. 5

1.18 QC RAD (Quality Control and Continuity of Surface Radiation Measurements)..................... 5

1.19 QME AERI LBL (Quality Measurement Experiment AERI Line-by-Line) .............................. 6

1.20 QME AERI LBL Clouds (QME AERI Line-by-Line Clouds) ............................................ 6

1.21 QME AERI PROF (Comparison of AERI profiles with radiosondes) ..................................... 6

1.22 QME LW FLUX (QME Longwave FLUX) ................................................................... 7

1.23 QME MWR COL (QME Microwave Radiometer Column)................................................... 7

1.24 QME MWR PROF (QME Microwave Radiometer Profiles) ................................................ 7

1.25 RLPROF ASR (Raman Lidar Profiles - Aerosol Scattering Ratio) .......................................... 7

1.26 RLPROF BE (Raman Lidar Profiles - Best Estimate) ….................................................... 8

1.27 RLPROF DEP (Raman Lidar Profiles - Depolarization) …................................................ 8

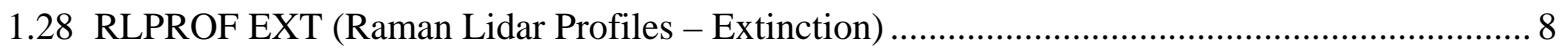

1.29 RLPROF MERGE (Raman Lidar Profiles - Merge) .......................................................... 8

1.30 RLPROF MR (Raman Lidar Profiles - Mixing Ratio) .......................................................... 9

1.31 SFC CLD GRID (SGP Area Surface Cloud and SW Radiation Grid) ...................................... 9

1.32 SW DIFF CORR (Shortwave Diffuse Correction) ............................................................. 9

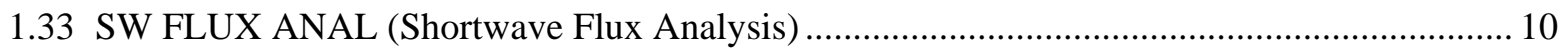

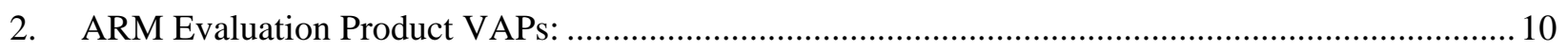

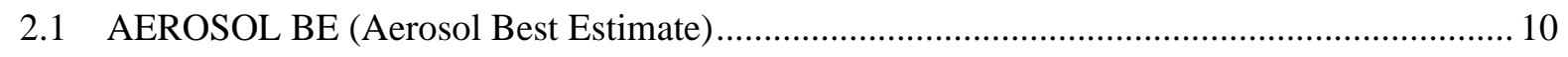

2.2 BBHRP (Broadband Heating Rate Profile)........................................................................ 10

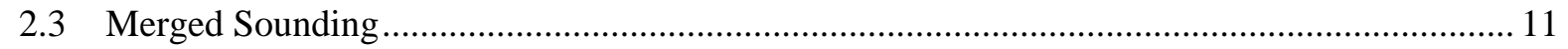

2.4 MICROBASE (Continuous Baseline Microphysical Retrieval)............................................ 11

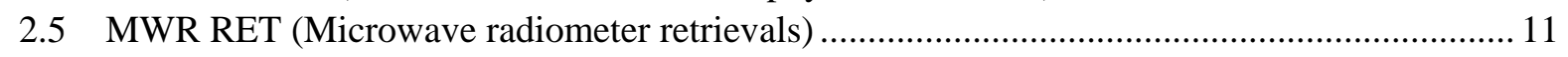

2.6 VAR ANAL (Variational Analysis Products) ...................................................................... 12 


\section{Current Production VAPs}

\subsection{Atmospherically Emitted Radiance Interferometer (AERI) Noise Filter}

Description: The original temporal resolution of the Atmospheric Radiation Measurement (ARM) AERI instruments was 8 minutes, where each cycle consisted of a 3-minute sky-view period and 2-minute views at each of the two blackbody targets. This sampling strategy was chosen to achieve the desired signal-tonoise ratio for clear-sky spectroscopy and profiling studies. To make the AERI observations more useful for cloud research, the temporal resolution has been decreased by an order of magnitude; however, this greatly increases the random error in these observations. This VAP uses a principle component analysis noise filter to significantly reduce the amount of uncorrelated random error in the AERI observations. The noise-filtered "rapid-sample" AERI observations have approximately the same amount of random error as the original AERI radiance data.

Working Group (WG)/Science Sponsor: Radiative Processes Working Group (RPWG)/Dave Turner and Bob Knuteson

Translator: Dave Turner

Status: Runs in batch mode, approximately every 2 weeks, for the Southern Great Plains (SGP) Central Facility (CF) and North Slope of Alaska (NSA) Barrow AERIs. Data are current unless otherwise noted. VAP will be applied to Tropical Western Pacific (TWP) Nauru and Darwin and ARM Mobile Facility (AMF) AERIs, as those AERIs migrate to rapid-sample operation.

\subsection{AERI PROF (AERI Profiles)}

Description: High temporal resolution temperature and water vapor profiles through the planetary boundary layer are retrieved from high-resolution spectral data observed by the AERI.

WG/Science Sponsor: RPWG/Wayne Feltz

Translator: Jennifer Comstock

Status: Runs daily for the SGP CF. Data are current unless otherwise noted. Currently runs on original AERI-01 instrument and is being modified to run for newer model AERI instruments at SGP and other sites.

\subsection{AIP (Aerosol Intensive Properties)}

Description: The AIP VAP computes several aerosol intensive properties. Intensive properties are independent of aerosol amount, depending only on the nature of the specific aerosol. The intensive properties of hygroscopic growth factor, aerosol single-scattering albedo, hemispheric backscatter fraction, and angstrom exponent are produced at a 1-minute sample rate. The submicron scattering and absorption fraction are produced on an hourly sample rate. This data product is a required input for the Aerosol Observing System (AOS) Corr and AOS Fit Relative Humidity (RH), precursors to the Aerosol Best Estimate (ABE) and Broadband Heating Rate Profile (BBHRP).

WG/Science Sponsor: Aerosol Working Group (AWG)/John Ogren

Translator: Connor Flynn

Status: Runs daily for SGP and AMF. Data are current unless otherwise noted. Stopped, requiring maintenance. Last available data 30 September 2005. 


\subsection{AOS Fit RH (Aerosol Observing System Fit RH)}

Description: The AOS Fit RH VAP computes the aerosol scattering humidification factor from dry and humidified aerosol scattering data generated by the AOS Corr VAP. It is a direct input for ABE, a precursor to BBHRP.

WG/Science Sponsor: AWG/Ogren

Translator: Connor Flynn

Status: Runs daily for SGP and AMF. Data are current unless otherwise noted.

Stopped, requiring re-release.

SGP: Last available data are from 30 June 2006.

AMF: Point Reyes (PYE) and Niamey (NIM) deployments are fully processed.

\subsection{ARSCL (Active Remote Sensing of Cloud Layers)}

Description: The ARSCL VAP combines data from multiple active remote sensing instruments to produce an objective determination of cloud hydrometeor height distributions and estimates of their radar reflectivities, vertical velocities, and Doppler spectral widths, which are optimized for accuracy. These data provide fundamental information for retrieving cloud microphysical properties and assessing the radiative effects of clouds on climate. Much of the algorithm's calculations deal with merging and optimizing data from the radar's sequential operating modes, which have different advantages and limitations.

WG/Science Sponsor: Cloud Properties Working Group (CPWG)/ Eugene Clothiuax

Translator: Michael Jensen

Status: Runs in batch mode at Brookhaven National Laboratory (BNL). Data available via the ARM Data Archive for the following time periods:

- $\quad$ SGP - November 1996 - June 2006

- $\quad$ NSA - March 1998 - September 2006

- $\quad$ TWP C1 - July 1999 - December 2004, October 2006 - December 2006

- $\quad$ TWP C2 - November 1998 - November 2005, October 2006 - December 2006

- $\quad$ TWP C3 - January 2006 - August 2006.

\subsection{BAEBBR (Bulk Aerodynamics Energy Balance Bowen Ratio)}

Description: The BAEBBR VAP provides a best-estimate of sensible and latent heat fluxes from energy balance Bowen ratio (EBBR) station measurements and bulk aerodynamics calculations. The BAEBBR VAP calculates the bulk aerodynamic latent and sensible heat fluxes from EBBR station meteorological measurements and uses these in place of the EBBR flux measurements when the Bowen ratio is between 1.6 and -0.45 . The resultant data set is considered to provide the "best estimate" of the diurnal cycle of fluxes.

WG/Science Sponsor: Cloud Modeling Working Group (CMWG)/David Cook Translator: Shaocheng Xie

Status: Run daily. Data are current unless otherwise noted, for all SGP sites with EBBR data. It is not running due to errors found in the BAEBBR VAP programming. The old code used fixed automated exchange mechanism (AEM [top; $1.9 \mathrm{~m}$ and bottom; $0.9 \mathrm{~m}$ ]) and wind speed sensor heights to generate the BAEBBR VAP, instead of site specific heights (which have ranged from 1.5 to 3.11 for the top height). This problem requires a complete re-run of the VAP program for the many years of EBBR data 
(from the start of the keeping of vegetation height). Estimated time for resumed operations is around the summer 2007. Data are available from 1 October 1995 to 30 June 2003.

\subsection{BEFLUX (Best-estimate Surface Radiative Flux)}

Description: The BEFLUX VAP processes data from three SGP CF radiometer systems when all are available. Before 2001, this VAP used corrected diffuse shortwave from the DiffCorr1Dutt VAP as input. The 1-minute input data are compared to decide which to use for averaging to get the best estimate of all surface radiative energy budget terms (shortwave and longwave downwelling and upwelling). The output data are saved in two netCDF files containing the best estimate values, QC flags, and the difference fields. WG/Science Sponsor: RPWG/Chuck Long

Translator: Chuck Long

Status: Runs for the SGP CF only. Data are current unless otherwise noted.

\subsection{IAP (In-Situ Aerosol Profile)}

Description: The IAP VAP applies corrections to instrument-level in situ aerosol profile data to yield calibrated, quality-assessed extensive aerosol properties along level flight legs.

WG/Science Sponsor: AWG/John Ogren

Translator: Connor Flynn

Status: Runs periodically in response to irregular flight schedules, generally monthly, only at SGP CF. Stopped. Needs maintenance to accommodate new instrument content. Last available data are from 30 September 2006.

\subsection{LANGLEY (Langley regression)}

Description: This VAP computes Langley regressions from the multi-filter rotating shadowband radiometer (MFRSR) and normal incidence multi-filter radiometer (NIMFR) direct beam solar irradiance measurements. The results of the Langley regressions are ultimately used as the basis for calibrations of these instruments via the MFRSROD1 Mich VAP.

WG/Science Sponsor: AWG/Joseph Michalsky

Translator: Connor Flynn

Status: Runs daily at all sites with MFRSRs or NIMFRs. Data are current unless otherwise noted.

\subsection{LBL CLOUD E (Line-by-Line Cloud Emissivity)}

Description: The LBL CLOUD E computes high-spectral resolution infrared cloud emissivity spectrum from AERI observations and clear-sky calculations from the LBLRTM. The original purpose was to develop a "climatology" of cloud emissivity for inclusion in fast radiative transfer models.

WG/Science Sponsor: RPWG/Tony Clough

Translator: Chuck Long

Status: Stopped, needs maintenance (need to update the LBLRTM to a more current version). Last available data are from 10 August 1998. This VAP has been recommended as a candidate for sunsetting.

\subsection{LBL RTM AERI (Line-by-Line Radiative Transfer Model to match AERI)}

Description: This VAP computes downwelling high-spectral resolution clear-sky infrared radiance with the line-by-line radiative transfer model (LBLRTM) and convolves it with the instrument response 
function of the AERI. This is a critical input for the QME AERI/LBLRTM VAP, which statistically compares the AERI observations and LBLRTM calculations.

WG/Science Sponsor: RPWG/Tony Clough

Translator: Chuck Long

Status: Stopped, needs maintenance (need to update the LBLRTM to a more current version). Last available data are from 8 January 2005. This VAP has been recommended as a candidate for sunsetting.

\subsection{LBL MWR (Line-by-line calculations compared to the MWR)}

Description: This VAP uses the radiative transfer model "monoRTM," which is a monochromatic version of the AERI LBLRTM to compute downwelling radiance at the two frequencies observed by the MWR (23.8 and 31.4 GHz). Thus, this VAP, facilitates the comparison between the observations and calculations.

WG/Science Sponsor: RPWG / Tony Clough

Translator: Dave Turner

Status: Runs for SGP, NSA, and TWP central facilities. Stopped, needs maintenance (need to update the LBLRTM to a more current version). Last available data are from 5 July 2004. This VAP has been recommended as a candidate for sunsetting.

\subsection{LS SONDE (Liebe Scaled Sonde)}

Description: This VAP generates radiosonde profiles where the RH profile is scaled to match the microwave radiometers (MWRs) precipitable water vapor (PWV). Because the model used in the MWRLOS product has been changed to monoRTM, this VAP is now inappropriately named.

WG/Science Sponsor: CPWG/ Tony Clough

Translator: Michael Jensen

Status: Runs operationally for the SGP CF daily. Data are current unless otherwise noted. Historical data are available [16 June 1997 - 8 October 2000] for four boundary sites.

\subsection{MFRSR CLDOD (Cloud Optical Properties)}

Description: The MFRSR CLDOD VAP generates cloud optical properties (optical depth and effective radius) using MFRSR diffuse observations from 415

nanometers, an MWR, and the Langley analysis VAP (Langley VAP). The retrievals are only valid for overcast liquid water clouds.

WG/Science Sponsor: CPWG/Qilong Min

Translator: Jennifer Comstock

Status: Runs daily at the SGP CF only. Data are current unless otherwise noted. Currently stopped because an input datastream (MFRSRLANGLEY) is undergoing maintenance. Last available data are from 24 November 2006.

\subsection{MFRSR OD (MFRSR Optical Depths)}

Description: This VAP has the following four important functions:

- generates robust calibrations for the MFRSR and NIMFR instruments from Langley analysis,

- applies this calibration to the irradiance data

- applies a moving filter cloud screen to identify cloud events 
- computes aerosol optical depths at five measured wavelengths with nominal centers at $415 \mathrm{~nm}$, $500 \mathrm{~nm}, 615 \mathrm{~nm}, 673 \mathrm{~nm}$, and $870 \mathrm{~nm}$.

WG/Science Sponsor: AWG/Joseph Michalsky

Translator: Connor Flynn

Status: Runs monthly at all sites with an MFRSR or NIMFR. Stopped. Maintenance finished, awaiting re-release. Last available data varies by site but generally not past December 2005.

\subsection{MPL NOR (MPL Normalized)}

Description: This VAP applies corrections to remove instrument artifacts from micropulse lidar (MPL) data, computes signal to noise levels, reports cloud-base height, and provides two cloud masks using robust and sensitive cloud detection algorithm.

WG/Science Sponsor: CWG and AWG/Campbell

Translator: Connor Flynn

Status: Runs daily for all central facilities, data are current unless otherwise noted. Stopped, needs maintenance. Changes to raw instrument data content and format require accommodation, but this is secondary to requiring new instrument corrections at all sites. Due to sensitivity of the algorithm to the systematic corrections (e.g., overlap), which are determined manually, this VAP will require periodic maintenance and some amount of reprocessing to apply updated corrections to the data after its initial processing. Last available data are from 11 May 2004.

\subsection{MWR AVG (Microwave Radiometer Averaging)}

Description: This VAP averages the principle fields from the MWR (23.8-GHz sky brightness temperature, 31.4-GHz sky brightness temperature, total water vapor along LOS path, and total liquid water along LOS path) over 1- and 5-minute intervals.

WG/Science Sponsor: Nancy Miller

Translator: Connor Flynn

Status: Runs daily. Data are current except at AMF where it has not been run. This VAP has been recommended as a candidate for sunsetting.

\subsection{QC RAD (Quality Control and Continuity of Surface Radiation Measurements)}

Description: The QC RAD VAP assesses data quality and enhances data continuity for the ARM surface broadband radiation data collected at all ARM facilities. The methodology uses climatological analyses of the surface radiation measurements to define reasonable limits for testing the data for unusual data values. Data that fall outside the normal range of occurrences are labeled either "questionable" or "bad," depending on how far outside the normal range the particular data reside. The methodology not only sets standard maximum and minimum value limits, but it includes many cross-comparisons based on how these instruments behave in the field in developing other VAPs, such as the Diffuse IR Loss Correction VAP and the BEFLUX VAP.

WG/Science Sponsor: RPWG/Chuck Long

Translator: Chuck Long

Status: Runs at all sites daily. Data are current unless otherwise noted. 


\subsection{QME AERI LBL (Quality Measurement Experiment AERI Line-by-Line)}

Description: Observed radiances as measured by the AERI and LBLRTM calculated radiances are produced for comparison. The three principle components of this study include the following:

- ground-based measurements of spectral radiances

- characterization of the atmospheric state associated with the radiating column

- evaluation of the LBLRTM, with the initial focus on clear sky.

In addition to providing statistics over the entire spectral band from 3.3-18 cm-1 (the range of the AERI), and statistics for each channel, the entire spectrum is divided into 17 spectral regions called "bins." These bins are disjoint intervals such that the union of them covers the entire spectrum selected to match the spectral bands for rapid radiation models in general circulation models (GCMs). To further facilitate analysis, each spectral element was associated with a specific physical process.

WG/Science Sponsor: RPWG/ Tony Clough

Translator: Chuck Long

Status: Stopped, needs maintenance (need to update the LBLRTM to a more current version). Last available data are from 8 January 2005. This VAP has been recommended as a candidate for sunsetting.

\subsection{QME AERI LBL Clouds (QME AERI Line-by-Line Clouds)}

Description: The QME AERI LBL Clouds compares of clouds data from the AERI versus LBLRTM model runs. Data from the various ceilometers, imagers, in situ measurements, and passive sensors are gathered, then used to determine whether the column of sky directly above the site central facility is not clear Another goal of this platform is to capture the variability of the atmospheric column, i.e., how fast it is changing over time. A natural byproduct of this compilation of different datastreams all matched to the same time interval is the ability to easily compare datastreams. For instance, the cloud-base height as measured by the Belfort laser ceilometer, theMPL, and a simple estimation derived from the AERI using nearby radiosonde temperature profiles can be compared. In addition, data between the infrared thermometer (IRT) and the AERI can be compared where the AERI data has been integrated and a filter function applied to match the IRT's spectral response.

WG/Science Sponsor: RPWG/ Tony Clough

Translator: Chuck Long

Status: Runs daily at all sites daily, data are current unless otherwise noted. This VAP has been recommended as a candidate for sunsetting.

\subsection{QME AERI PROF (Comparison of AERI profiles with radiosondes)}

Description: This VAP routinely compares the retrieved temperature and water vapor profiles from the AERIPROF VAP with coincident radiosonde profiles.

WG/Science Sponsor: RPWG/Wayne Feltz

Translator: Jennifer Comstock

Status: Runs daily at the SGP CF only. Data are current unless otherwise noted. Currently stopped and undergoing maintenance. Last available data are from 26 January 2004. This VAP has been recommended as a candidate for sunsetting. 


\subsection{QME LW FLUX (QME Longwave FLUX)}

Description: The QME LW FLUX compares longwave flux data from the AERI, pyrgeometer, rapid radiative transfer model (RRTM), and LBLRTM. The AERI measures downwelling spectral radiance at high spectral resolution from 3.3 - $18 \mu \mathrm{m}$; these observations are accurate to better than $1 \%$ due to frequent views of two well-characterized blackbody targets. The pyrgeometer measures downwelling flux from 3-50 $\mu \mathrm{m}$; however, this instrument in calibrated only once per year. This VAP uses the LBLRTM to extend the AERI observations from 18 - $50 \mu \mathrm{m}$, then compute the wavelength-dependent factors needed to convert the high-spectral-resolution radiance into flux to compare the AERI and the pyrgeometer. Additionally, the longwave flux is computed from the LBLRTM and the RRTM for intercomparisons. WG/Science Sponsor: RPWG/Tony Clough

Translator: Chuck Long

Status: This VAP runs for the SGP CF only. Stopped, needs maintenance. Last available data are from 16 April 2003. This VAP has been recommended as a candidate for sunsetting.

\subsection{QME MWR COL (QME Microwave Radiometer Column)}

Description: The QME MWR COL compares the sonde profile of water vapor to MWR data from +/- 20 minutes before the balloon launch time. Both the PWV and brightness temperatures are compared. For the latter, an out-dated model is used for the MWR data.

WG/Science Sponsor: RPWG/Jim Liljegren

Translator: Ric Cederwall

Status: Runs at SGP, TWP, and NSA CFs. Retired. Functionality replaced by MWRRET VAP. Last available data are from 5 July 2004. This VAP has been recommended as a candidate for sunsetting.

\subsection{QME MWR PROF (QME Microwave Radiometer Profiles)}

Description: This VAP routinely computes statistics (bias, RMS) between the MWRPROF profiles of temperature and humidity and radiosondes.

WG/Science Sponsor: RPWG/Ed Westwater

Translator: Chuck Long

Status: Retired. Functionality replaced by MWRRET VAP. Last available data are from 5 July 2004. This VAP has been recommended as a candidate for sunsetting.

\subsection{RLPROF ASR (Raman Lidar Profiles - Aerosol Scattering Ratio)}

Description: The RLPROF ASR VAP computes the calibrated aerosol scattering ratio (ASR) and backscatter coefficient profiles from the Raman lidar data.

WG/Science Sponsor: AWG/Rich Ferrare

Translator: Jennifer Comstock

Status: Runs daily at the SGP CF only. Data are current unless otherwise noted. RLPROFASR has been updated to interface with new RLPROFMERGE data. This VAP will not be turned back on until RLPROFMERGE is released. Last available data are from 6 January 2004. 


\subsection{RLPROF BE (Raman Lidar Profiles - Best Estimate)}

Description: The RLPROF BE VAP combines the output from all other RLPROF VAPs and interpolates the profiles to a common vertical and temporal resolution. Output profiles include aerosol scattering ratio, backscatter, extinction, water vapor mixing ratio, and depolarization ratio.

WG/Science Sponsor: AWG and CPWG/Dave Turner

Translator: Jennifer Comstock

Status: Runs daily at the SGP CF only. Data are current unless otherwise noted. RLPROFBE is stopped until maintenance of input VAPs are completed. Last available data are from 6 January 2004.

\subsection{RLPROF DEP (Raman Lidar Profiles - Depolarization)}

Description: The RLPROF DEP VAP computes depolarization ratio profiles and cloud optical depth using Raman lidar data.

WG/Science Sponsor: CPWG/Dave Turner

Translator: Jennifer Comstock

Status: Runs daily at the SGP CF only. Data are current unless otherwise noted. RLPROFDEP is stopped and undergoing maintenance to interface with the new RLPROFMERGE input files. This VAP will not be turned back on until RLPROFMERGE is released. Last available data are from 6 January 2004.

\subsection{RLPROF EXT (Raman Lidar Profiles - Extinction)}

Description: The RLPROF EXT VAP computes aerosol extinction profiles using Raman lidar data and the profile of extinction to backscatter ratio. Aerosol optical depth is also computed.

WG/Science Sponsor: AWG/Rich Ferrare

Translator: Jennifer Comstock

Status: Runs at daily at the SGP CF only. Data are current unless otherwise noted. RLPROFEXT is stopped and undergoing maintenance to interface with the new RLPROFMERGE input files. This VAP will not be turned back on until RLPROFMERGE is released. Last available data are from 6 January 2004.

\subsection{RLPROF MERGE (Raman Lidar Profiles - Merge)}

Description: This VAP merges the analog and photon counting channels measured by the Raman lidar. These "merged" profiles are then input to all other RLPROF VAPs. This VAP is required after the Raman lidar was upgraded in September 2004; data collected before this period will not be processed with this code.

WG/Science Sponsor: AWG/David Turner

Translator: Jennifer Comstock

Status: Runs daily at the SGP CF only. Data are current unless otherwise noted. RLPROFMERGE is stopped and undergoing maintenance to understand the diurnal cycle of the coefficients required for merging the analog and photon counting channels. Erroneous data removed from ARM Data Archive, no data available. 


\subsection{RLPROF MR (Raman Lidar Profiles - Mixing Ratio)}

Description: This VAP computes water vapor mixing ratio profiles using Raman lidar data and RH profiles. It uses MWR lines-of-sight (LOS) PWV data as the calibration source.

WG/Science Sponsor: CPWG, AWG, and RPWG/Dave Turner

Translator: Jennifer Comstock

Status: Runs daily at the SGP CF only. Data are current unless otherwise noted. The RLPROFMR VAP is stopped and undergoing maintenance to interface with the new RLPROFMERGE input files. This VAP will not be turned back on until RLPROFMERGE is released. Last available data are from 6 January 2004.

\subsection{SFC CLD GRID (SGP Area Surface Cloud and SW Radiation Grid)}

Description: The SFC CLD GRID VAP uses as input the 15-minute output from the SW FLUX ANAL VAP from the SGP CF and extended Facilities. It applies a multi-pass weighted sum analytic approximation technique that uses Gaussian weighting and an imposed scale length, to interpolate to a 0.25 degree by 0.25 degree latitude/longitude grid over the SGP domain. The output, like the input, includes only solar elevation angles of 10 degrees or greater, producing gridded output for cloud fraction, the ratio of measured over clear-sky short wave total (both unshaded pyranometer and sum of direct and diffuse) and direct irradiance, the estimated clear-sky fit total shortwave irradiance, and the estimated clear-sky fit direct shortwave irradiance. The ratio measured over clear-sky direct shortwave, in conjunction with the total shortwave ratio and clear-sky total and direct irradiance, allows for component evaluation of the irradiance and cloud effects, as well as climatological and statistical research.

WG/Science Sponsor: RPWG/Chuck Long

Translator: Chuck Long

Status: Runs monthly at all SGP facilities. Data are current, 1-2 months after data collection, unless otherwise noted.

\subsection{SW DIFF CORR (Shortwave Diffuse Correction)}

Description: The SW DIFF CORR VAP corrects diffuse shortwave measurements for infrared loss. The DIFFCORR1DUTT VAP uses two techniques to correct shortwave data during daytime hours, using information from a collocated pyrgeometer. Both techniques collect nighttime data with corresponding pyranometer infrared-loss data, and separately fit for two modes: dry and moist. Each mode is detected separately in the nighttime data and applied separately during the daytime as appropriate. The DiffCorr1Dutt VAP output files contain a best estimate of the downwelling shortwave, and a best estimate of the diffuse shortwave. In addition, the output files contain other useful values, such as an estimate of the clear-sky Rayleigh diffuse shortwave amount, and standard surface meteorological measurements. Because ARM stopped using shaded Eppley PSPs in 2001, using the Eppley model 8-48 "Black and White" pyranometers that do not have a significant infrared loss problem instead, this VAP includes a "retirement" end date for each ARM facility from the start.

WG/Science Sponsor: RPWG/ Ells Dutton/

Translator: Chuck Long

Status: Due to nature of methodology, requires manual processing on a yearly basis.

All ARM shaded PSP data has been corrected for infrared loss with the exception of NSA, where inclusion of heaters in the radiometer ventilators has cast doubt on the applicability of the methodology under those circumstances. Results of the current NSA Radiometer field campaign will be used to 
investigate the applicability and refine anddevelop methodology as needed for quantifying and correcting for infrared loss with heated ventilation. For SGP and TWP data have been retired.

\subsection{SW FLUX ANAL (Shortwave Flux Analysis)}

Description: The SW FLUX ANAL VAP applies a clear-sky detection and fitting technique to data from broadband shortwave radiometers located at all ARM facilities. This technique uses hemispheric, broadband total and diffuse shortwave irradiance measurements to identify clear-sky periods using the known characteristics of typical clear-sky irradiance time series. These clear-sky estimates are then used to fit functions for clear periods, interpolate the fit coefficients for cloudy periods, and produce a continuous estimate of clear-sky shortwave to infer the effect of cloudiness on the measured downwelling shortwave at 1-minute resolution for daylight hours. The 1-minute resolution analysis also includes data quality assessment that goes beyond standard min/max/delta and component comparison testing. The SW FLUX ANAL VAP also uses a technique to infer average fractional sky cover at 15-minute resolution for solar elevation angles 10 degrees or greater, which is used as input for the SFC CLD GRID VAP.

WG/Science Sponsor: RPWG/ Chuck Long

Translator: Chuck Long

Status: Runs monthly at all ARM facilities, except for Darwin, Australia, which requires manual processing. Data are current, 1-2 months after data collection, unless otherwise noted (i.e., Darwin).

TWR MR (Tower Water-Vapor Mixing Ratio)

Description: The purpose of this algorithm is to calculate water-vapor mixing ratio at the 25- and 60-m levels of the tower at the SGP CF.

WG/Science Sponsor: AWG and CPWG/David Turner

Translator: Jennifer Comstock

Status: Currently running on the Data Management Facility (DMF) for SGP only. Runs daily. Data are current unless otherwise noted.

\section{ARM Evaluation Product VAPs:}

\subsection{AEROSOL BE (Aerosol Best Estimate)}

Description: The AEROSOL BE VAP provides temporally and spatially continuous vertical profiles of ambient aerosol optical properties including scattering, absorption, and extinction coefficients, single scattering albedo and asymmetry parameter. It uses a combination of passive radiometers, in-situ surface measurements, empirical relationships, climatologies, and model input. It is a direct input to the BBHRP VAP, an ACRF programmatic metric.

WG/Science Sponsor: AWG/Dave Turner

Translator: Connor Flynn

Status: This product has been produced in batch mode for BBHRP milestones at SGP for 2000 and at NSA for 2004. A version will be provided for BBHRP TWP efforts.

\subsection{BBHRP (Broadband Heating Rate Profile)}

Description: The BBHRP VAP employs output from several different VAPS defining profiles of the atmospheric state, cloud microphysical properties, aerosol properties and surface characteristics as input 
to a state-of-the-art radiative transfer model. The output of this VAP are profiles of broadband (shortwave and longwave) radiative heating rates.

WG/Science Sponsor: RPWG and CPWG/Eli Mlawer

Translator: Michael Jensen and Chuck Long

Status: A series of BBHRP trials have been performed for the SGP (March 2000 February 2001) and NSA (March 2004 August 2004) sites. Current efforts are aimed at improving the input streams at the NSA.

\subsection{Merged Sounding}

Description: The Merged Sounding VAP uses a combination of observations from radiosonde soundings, the MWR, surface meteorological instruments, and European Centre for Medium Range Weather Forecasts (ECMWF) model output with a sophisticated scaling/interpolation/smoothing scheme in order to define profiles of the atmospheric thermodynamic state at 1 minute intervals and a total of 266 altitude levels.

WG/Science Sponsor: CPWG / Jay Mace

Translator: Michael Jensen

Status: Runs at BNL in batch mode. The following data are available:

- $\quad$ SGP - complete for 1 year (March 2000 - February 2001)

- NSA - January 2004 - December 2004

- $\quad$ TWP - Manus February 2000 - July 2000, October 2004; Nauru March 1999 - December 1999, October 2004; Darwin October 2004, January 2005.

\subsection{MICROBASE (Continuous Baseline Microphysical Retrieval)}

Description: The MICROBASE VAP uses a combination of observations from the MMCR, ceilometer, MPL, MWR and balloon-borne sounding profiles in order to determine the profiles of liquid/ice water content liquid/ice cloud particle effective radius and cloud fraction. This baseline retrieval is calculated every 10 seconds and then averaged over 20-minute intervals with a vertical resolution of greater than 230 levels.

WG/Science Sponsor: CPWG/ Mark Miller

Translator: Michael Jensen

Status: The MICROBASE VAP is currently operational at BNL in batch mode. The following data are available:

- $\quad$ SGP - March 2000 February 20/01

- $\quad$ NSA prototype - January 2004 - December 2004

- $\quad$ TWP-C1 - November 2003 - October 2004.

\subsection{MWR RET (Microwave radiometer retrievals)}

Description: This VAP provides PWV and liquid water path (LWP) from the 2-channel MWR using two advanced retrieval algorithms: a physical-iterative method and a variable-coefficient statistical method. Both methods use the monoRTM as the forward model. Additionally, the VAP applies small $(<1 \mathrm{~K})$ offsets to the observed brightness temperatures to reduce the systematic error in the observations and the forward model; these biases are computed directly from the observations. The temperature of the cloud liquid is also accounted for in the retrievals used in this VAP.

WG/Science Sponsor: CPWG and RPWG/Jim Liljegren and Tony Clough 
Translator: Dave Turner

Status: Currently operated manually, with data available for multiple years of data from most of the ARM sites as an ARM evaluation product. VAP will be migrated to automated operation in the near future.

\subsection{VAR ANAL (Variational Analysis Products)}

Description: The large-scale forcing and evaluation datasets for single-column models and cloudresolving models. The data were produced from the ARM sounding data (during ARM field campaigns) or the rapid update cycle analysis data (for the continuous period 1999-2001) constrained with the ARM ground measurements and the NOAA/NASA satellite data using the variational analysis method. This VAP depends on data from other VAPs, such as BEFLUX and BAEBBR. The output data are saved in both netCDF files and ASCII files containing both the single-column models domain-averaged forcing data (e.g., the large-scale vertical velocity and advective tendencies of temperature and moisture) and model evaluation data (e.g., surface and top of the atmosphere measurements).

WG/Science Sponsor: CMWG/Minghua Zhang

Translator: Shaocheng Xie

Status: Requires manual operation. Available for all ARM SGP field campaigns and the continuous period 1999-2001, 2004 Mixed-Phase Arctic Cloud Experiment (M-PACE) field campaign at the NSA site. 2006 Tropical Warm Pool - International Cloud Experiment (TWP-ICE) field campaign variational analysis data will be available by the end of March 2007. 